\title{
Analysis of PID Control Effect for Nonlinear Systems with Related High Order Bounded Unknown Functions
}

\author{
Yafei $\mathrm{Li}^{1}$, Dingxiong Zhang ${ }^{1}$, Yidong Wang ${ }^{1,}$ a, Hong Wang ${ }^{2}$ and Junwei Lei ${ }^{2}$ \\ 1The $91872^{\text {th }}$ unit of PLA, Beijing, China \\ ${ }^{2}$ Department of control engineering, Naval aeronautical and Astronautical University Yantai, 264001 \\ awangyidong96107@163.com
}

Keywords: Related order, bounded, nonlinearity, stability, numerical simulation

\begin{abstract}
According to the nonlinear function of relative power function order, the uncertainty function is classified as one order relatively bounded and two order relative bounded and higher relative bounded situation. Then in view of the above three categories of uncertain nonlinear system, PID control law is designed by choosing Lyapunov function, the stability of the system is analyzed, and the relationship of PID parameters and the system stability is proposed, and also the system anti constant interference ability is studied.
\end{abstract}

\section{Introduction}

Nonlinearity and uncertainty have always been the two difficult problems in the field of control theory[1-4]. And PID control algorithm is the most widely used algorithm in industry and military applications. And for the measurement of the degree of nonlinearity, there is no good theory to measure[5-7]. Considering that we can distinguish the control complexity of the nonlinear function according to the order of its power function. Therefore, according to the order of nonlinear functions, nonlinear systems are divided into a relative one order, relative to the second order and relative high order situation[8]. and respectively in accordance with the PID control law design, stability analysis, and the anti-interference ability for the constant interference of the system are studied. Finally, the relationship between stability and control parameters selection is given, which is very meaningful to control engineers.

\section{Design of the Chip Kick Mechanics}

To make the main idea of this paper more easy to understand, a kind of simple first order nonlinear system is taken as an example:

$$
\frac{\sqrt{l^{2}+w^{2}}}{\sin (\phi+\theta)}=\frac{r}{\sin \theta}=\frac{R}{\sin \phi}
$$

Where $x$ is system state, and $F$ is constant disturbance.

And the control object is to design a controller such that the system state can track the desired value $x^{d}$. But the main purpose of this paper is to show the performance of PID controller when the nonlinear system is affected by constant disturbance and how to calculation related specifications.

So the complex problem is divided into three situations. First situation is that the nonlinear system is bounded as follows.

\section{Analysis of PID Control for Bounded Nonlinear System with Constant Disturbance}

First situation is that we assume the nonlinear function in system model is bounded, then it holds

$$
|f(x)|<M
$$

Then we can design a bigger gain such that $k_{w}=k_{p}<0$ to make the system stable. And the 
nonlinear function can be viewed as a part of constant disturbance $F$.

To make the analysis more, we use Lyapunov function method to prove it. Then the below error system can be written as

$$
\dot{e}=f(x)+k_{p} e+k_{i} \int e d t+k_{d} \dot{e}+F
$$

And it can be transformed as

$$
e\left(1-k_{d}\right) \dot{e}-k_{i} e \int e d t=k_{p} e^{2}+(F+f(x)) e
$$

Define a Lyapunov function

$$
V=\frac{1}{2\left(1-k_{d}\right)} e^{2}+\frac{-k_{i}}{2}\left(\int e d t\right)^{2}>0
$$

Then solve its derivative as

$$
\dot{V}=k_{p} e^{2}+(F+f(x)) e
$$

So because of the boundedness of nonlinear function, define $|F+f(x)| \leq M_{f}$, then the above system can be stable for a small $k_{p}$, but there may be steady state error exists.

\section{Analysis of PID Control for Related First Order Bounded Nonlinear System with Constant Disturbance}

So the second situation is considered for the related nonlinear systems. Then we assume nonlinear function satisfied $|f(x)|<M_{1}|x|+M_{2}$, and design a PID control law as

$$
\dot{e}=f(x)+k_{p} e+k_{i} \int e d t+k_{d} \dot{e}+F
$$

Since $M_{1}|x| \leq M_{1}\left|x^{d}\right|+M_{1}|e|$, then

$$
e \dot{e} \leq k_{p} e e+k_{i} e \int e d t+k_{d} \dot{e} e+\left(F+M_{1}\left|x^{d}\right|+M_{2}\right)|e|+M_{1}|e|^{2}
$$

And define a Lyapunov function as

$$
V=\frac{1}{2\left(1-k_{d}\right)} e^{2}+\frac{-k_{i}}{2}\left(\int e d t\right)^{2}>0
$$

Then it holds

$$
\dot{V} \leq\left(k_{p}+M_{1}\right) e^{2}+\left(F+M_{1}\left|x^{d}\right|+M_{2}\right)|e|
$$

Then the system is stable if $k_{p}+M_{1}<0$. Also there is steady state error according to the proof process. But the below simulation result shows that there is no steady state error, so the above proof is conservative. The below simulation is done with a example that $f(x)=\sin x+3 x+x^{2 / 3}$, and the simulation result is shown as below figure 1 .

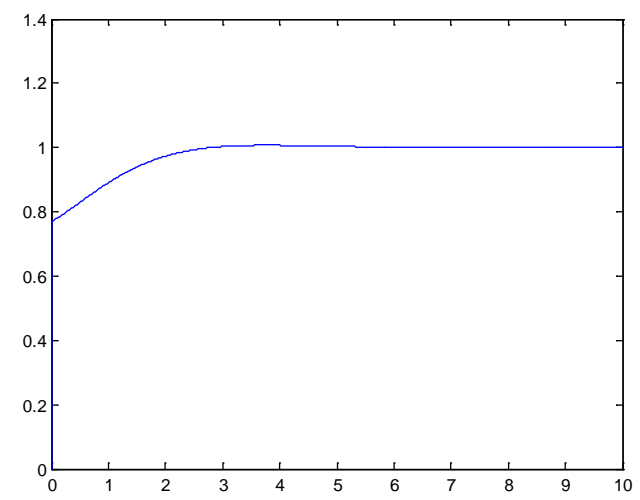

Fig.1. The curve of state $\mathrm{x}$

Where the derivative is solved with a filter with a time delay constant $0.01 \mathrm{~s}$. 


\section{Analysis of PID Control for Related Second Order Bounded Nonlinear System with Constant Disturbance}

The third situation is considering the system is second order bounded as $|f(x)|<M_{2} x^{2}+M_{1} x+M_{0}$, also design a PID control law as

$$
\dot{e}=f(x)+k_{p} e+k_{i} \int e d t+k_{d} \dot{e}+F
$$

Where

$$
e \dot{e} \leq k_{p} e e+k_{i} e \int e d t+k_{d} \dot{e} e+F|e|+M_{1} e^{2}+M_{2}\left|x^{d}\right|^{2}|e|+\left|M_{1} x_{1}^{d}\right||e|+M_{2}|e|^{2}|e|
$$

Also choose a Lyapunov function as

$$
\dot{V} \leq\left(k_{p}+M_{1}\right) e^{2}+\left(F+M_{1}\left|x^{d}\right|+M_{2}\left|x^{d}\right|^{2}\right)|e|+M_{2}|e|^{2}|e|
$$

Then the system is stable and bounded, and its stable interval can be calculated as

$$
\left(k_{p}+M_{1}\right) e^{2}+M_{2}|e|^{2}|e|<0(14)
$$

Then

$$
|e|<-\frac{\left(k_{p}+M_{1}\right)}{M_{2}}
$$

So we can make a conclusion that the proportion coefficient determine the system stable range.

So if the system is nonlinear and uncertain, it is meaningless to design a global stable control law, but if we design a bigger proportional coefficient, then the system has a big gain, and the system will has a bigger stable interval.

\section{Analysis of PID Control for Related High Order Bounded Nonlinear System with Constant Disturbance}

If we consider a high order related bounded situation for nonlinear system, such as

$$
|f(x)|<M_{3}\left|x^{3}\right|+M_{2} x^{2}+M_{1} x+M_{0}
$$

Then it holds:

$$
M_{3}\left|x^{3}\right|<M_{3}\left|e^{3}\right|+M_{3}\left|x^{d}\right|^{3}
$$

Use the same method, it also easy to get

$$
\dot{V} \leq\left(k_{p}+M_{1}\right) e^{2}+\left(F+M_{1}\left|x^{d}\right|+M_{3}\left|x^{d}\right|^{3}+M_{2}\left|x^{d}\right|^{2}\right)|e|+M_{2}|e|^{2}|e|+M_{3}|e|^{4}
$$

And the stable interval can be calculated as

$$
\left(k_{p}+M_{1}\right) e^{2}+M_{2}|e|^{2}|e|+M_{3}|e|^{4}<0
$$

Then it holds

$$
\left(k_{p}+M_{1}\right)+M_{2}|e|+M_{3}|e|^{2}<0
$$

It also shows that if the gain is bigger, then the system will has a bigger stable range.

\section{Conclusions}

Complex nonlinear systems are divided into related first order bounded system, related second order bounded system and related high order system three kinds of situation. Then PID controllers are designed according to above three situations respectively. Also the anti-disturbance ability is analyzed by using Lyapunov stability theorem and the parameters choosing method for PID controller is proposed, which is very meaningful for engineers to design a controller not only for simple first order systems but also for high order uncertain nonlinear systems. 


\section{References}

[1]Buschek $\mathrm{H}$ and Calise A J.Hypersonic flight control system design using fixed order robust controllers[A].In:AIAA Guidance,Navigation and Control Conference[C].Chattanooga,TN, AIAA 95-6062

[2]Buschek H, Calise A J. Uncertainty modeling and fixed-order controller design for a hypersonicvehicle model. Journal of Guidance,Control and Dynamics, 1997, 20(1): 42-48.

[3]Heller $\mathrm{M}$ and Sachs G.Flight dynamics and robust control of a hypersonic test vehicle with ramjet propulsion[A].In:AIAA International Space Planes and Hypersonic Systems and Technologies Conference[C].Norfolk,VA,AIAA 98-1521

[4]M. Ohno, Y. Yamaguchi, T. Hata, M. Takahama, Robust flight control law design for an automatic landing flight experiment [J], Control Engineering Practice, 1999, 7(9):1143-1152

[5]Naidu S D,Banda S S and Buffington J L.Unified approach to $\mathrm{H}_{2}$ and $\mathrm{H}_{\infty}$ optimal control of a hypersonic vehicle[A].In:Proceedings of the American control conference[C].San Diego, California,1999,vol.4:2737-2741.

[6] Christopher I. Marrison. synthesis of robust control systems for a hypersonic aircraft decision and contro1. IEEE, 1994。4:3324-3329

[7] E. mooij. 1inear quadratic regulator reentry control-performance assessment using a taguchi approach. AIAA, international space planes and hypersonic systems and technologies conference. 1998: 665-677.

[8]Kevin. P. Groves, Andrea. Serranti, Stephen. Yurkovich. Anti-Windup Control for an Air-breathing Hypersonic Vehicle Model. AIAA, 2006-6557: 1-14. 